\title{
Safety, Pharmacokinetics, and Clinical Activity of Adavosertib in Combination with Chemotherapy in Asian Patients with Advanced Solid Tumors: Phase Ib Study
}

\author{
Hidenori Kato ${ }^{1}$ Paul de Souza ${ }^{2,10}$. Sang-We Kim ${ }^{3}$. Jason D. Lickliter ${ }^{4}$ - Yoichi Naito ${ }^{5}$ Keunchil Park ${ }^{6}$. \\ Sanjeev Kumar ${ }^{7}$. Ganesh M. Mugundu ${ }^{8}$. Yung-Jue Bang ${ }^{9}$ (D)
}

Published online: 7 February 2020

(c) The Author(s) 2020

\begin{abstract} tumors and defined the recommended Phase II dose. performed and dose escalation/de-escalation conducted as appropriate. taxel and carboplatin in Asian patients.

\section{Introduction}

The tyrosine kinase WEE1 regulates cyclin-dependent kinase 1 (CDK1), which drives cells from the G2 phase into mitosis, and CDK2, which drives cells into and through the $\mathrm{S}$ phase of the cell cycle $[1,2]$. The tumor suppressor protein $\mathrm{p} 53$ is involved in regulation of the G1 checkpoint. Many human cancers have loss-of-function TP53 mutations, meaning that they become more dependent on the G2/M- and S-phase checkpoints to halt progression of the cell cycle $[3,4]$.
\end{abstract}

Background The WEE1 inhibitor adavosertib (AZD1775) has been investigated in Western patients.

Objective This open-label Phase Ib study (NCT02341456) investigated the safety, pharmacokinetics, and clinical activity of adavosertib in combination with carboplatin alone or paclitaxel plus carboplatin in Asian patients with advanced solid

Patients and methods Nineteen patients received adavosertib $175 \mathrm{mg}$ twice daily (bid) for 2.5 days (five doses) in combination with carboplatin (AUC 5) alone or paclitaxel $\left(175 \mathrm{mg} / \mathrm{m}^{2}\right.$ ) plus carboplatin, or adavosertib $225 \mathrm{mg}$ bid for 2.5 days in combination with paclitaxel plus carboplatin in 21-day cycles. Preliminary safety and dose-limiting toxicity analyses were

Results Adavosertib $175 \mathrm{mg}$ bid for 2.5 days with carboplatin alone or paclitaxel plus carboplatin was considered tolerable. Two patients receiving adavosertib $225 \mathrm{mg}$ bid in combination with paclitaxel plus carboplatin experienced dose-limiting toxicities (grade 4 sepsis; grade 5 acute respiratory distress syndrome); this regimen was not considered tolerable. Grade $\geq 3$ adverse events reported most commonly in any cohort included: anemia; decreased white blood cell count; decreased neutrophil count; neutropenia; decreased platelet count; thrombocytopenia; and febrile neutropenia. Exposure to adavosertib, as determined by pharmacokinetic analysis, in Asian patients was higher than that previously seen in Western patients. A partial response occurred in 2/12 evaluable patients (16.7\%) at the recommended Phase II dose.

Conclusions Adavosertib $175 \mathrm{mg}$ bid for 2.5 days was chosen as the recommended Phase II dose in combination with pacli-

Electronic supplementary material The online version of this article (https://doi.org/10.1007/s11523-020-00701-5) contains supplementary material, which is available to authorized users.

Yung-Jue Bang

bangyj@snu.ac.kr

Extended author information available on the last page of the article
Following DNA damage, WEE1 inhibits CDK1, leading to cell cycle arrest and allowing time for DNA repair [4]. Inhibiting WEE1 abrogates G2 cell cycle arrest, resulting in premature entry into mitosis and leading to aberrantly high CDK2 activity in S-phase cells, with the deregulated DNA replication resulting in replication stress [1,5]. WEE1 inhibition also exploits the G1 checkpoint deficiency seen in p53-deficient cells [3]. Thus, WEE1 inhibition sensitizes tumor cells to DNA-damaging chemotherapy and can lead to unstable DNA replication, DNA damage and mitotic catastrophe $[1,3]$.

Adavosertib (AZD1775) is a first-in-class, potent, selective WEE1 inhibitor. Adavosertib showed acceptable toxicity, linear pharmacokinetics, and target engagement when administered in combination with cisplatin, carboplatin, or gemcitabine in patients with advanced solid tumors in a Phase I study (NCT00648648, PN001) [6]. 


\section{Key Points}

Adavosertib $175 \mathrm{mg}$ twice daily (bid) for 2.5 days (five doses) in combination with carboplatin (AUC 5) alone or paclitaxel $\left(175 \mathrm{mg} / \mathrm{m}^{2}\right)$ plus carboplatin was considered tolerable in Asian patients with advanced solid tumors.

Exposure to adavosertib, as determined by pharmacokinetic analysis, was 30-45\% higher in Asian patients than that previously seen in Western patients.

In Asian patients, the recommended Phase II dose of adavosertib (175 $\mathrm{mg}$ bid for 2.5 days in combination with paclitaxel plus carboplatin) is lower than the recommended Phase II dose in Western patients, most likely reflecting the increased exposure of adavosertib in Asian patients.

In Phase II studies, adavosertib showed antitumor activity when administered in combination with carboplatin in women with TP53-mutated ovarian cancer refractory or resistant to first-line platinum-based chemotherapy (NCT01164995, PN009) [7], or in combination with paclitaxel plus carboplatin in women with TP53-mutated platinum-sensitive ovarian cancer (NCT01357161, PN004) [8]. In study PN004, progression-free survival was longer in patients receiving adavosertib with paclitaxel plus carboplatin than in those receiving placebo with paclitaxel plus carboplatin [8].

A Phase I study established a maximum tolerated dose of adavosertib of $225 \mathrm{mg}$ twice daily (bid) for 2.5 days (five doses; day 1, day 2, and the morning of day 3 ) per 21-day cycle when administered as monotherapy to Western patients with advanced solid tumors (NCT01748825) [9]. In Western patients, the recommended Phase II regimen of adavosertib for use in combination with paclitaxel $(175 \mathrm{mg} /$ $\mathrm{m}^{2}$ ) and carboplatin (AUC 5) is also $225 \mathrm{mg}$ bid for 2.5 days per 21-day cycle, which was used successfully in the PN004 study [8]. The aim of this Phase Ib study (NCT02341456) was to evaluate the safety and tolerability of the combination of adavosertib with carboplatin alone or with paclitaxel plus carboplatin in Asian patients with advanced solid tumors and to define the recommended Phase II dose. It is the first study to investigate this treatment regimen in this patient population.

\section{Methods}

\subsection{Patient Selection}

Eligibility criteria included patients who had histologic or cytologic confirmation of a locally advanced or metastatic solid tumor (excluding lymphoma), failure to respond to standard therapy, disease progression despite standard therapy, or cancers for which standard therapy did not exist. Patients also had to have at least one measurable lesion that could be accurately assessed at baseline by computed tomography or magnetic resonance imaging and Response Evaluation Criteria in Solid Tumors (RECIST) v1.1. Patients were required to have an absolute neutrophil count $\geq 1.5 \times 10^{9} / \mathrm{L}$, hemoglobin $\geq 90 \mathrm{~g} / \mathrm{L}$, platelets $\geq 100 \times 10^{9} / \mathrm{L}$, alanine aminotransferase and aspartate aminotransferase $\leq 2.5 \times$ the upper limit of normal (ULN) or $\leq 5 \times$ ULN if there were known hepatic metastases, and total bilirubin $\leq 1.25 \times$ ULN or total bilirubin $\leq 3.0 \times \mathrm{ULN}$ with documented Gilbert's syndrome (unconjugated hyperbilirubinemia). Patients who had undergone radiotherapy with a limited field of radiation for palliation within 1 week of the first dose of study treatment, with the exception of patients receiving radiation to $>30 \%$ of the bone marrow, or with a wide field of radiation within 4 weeks of the first dose of study treatment were excluded from the study, as were patients with refractory nausea and vomiting. Full eligibility criteria are summarized in the Supplementary Material.

\subsection{Study Design and Treatment}

This non-randomized, open-label, multicenter, Phase Ib study was conducted in seven centers in Australia, Japan, and South Korea.

Patients were initially recruited into cohorts 1 and 1a, with three to six patients planned for each cohort. Based on the data from earlier studies [6,10], patients in these cohorts received a single starting dose of oral adavosertib $175 \mathrm{mg}$ on day 1 (cycle 0) (Fig. 1 and Supplementary Material). This was followed $5 \pm 2$ days later by adavosertib $175 \mathrm{mg}$ bid for 2.5 days (five doses) in combination with intravenous paclitaxel (175 mg/m $\mathrm{m}^{2}$ as a 3-h infusion on day 1) plus intravenous carboplatin (AUC 5 on day 1) (cohort 1; A175PC) or carboplatin alone (AUC 5 on day 1) (cohort 1a; A175C) in subsequent 21-day cycles.

Preliminary safety and dose-limiting toxicity (DLT) analyses were performed and dose-escalation/de-escalation conducted as appropriate (Supplementary Material). A DLT was defined as an adverse event or abnormal laboratory value that occurred from the first dose of study treatment up to the last day of cycle 1 . Dose increases were permitted after review of data from a minimum of three evaluable patients. If no DLT was observed in a cohort of three to six evaluable patients, then dose escalation could occur. Based on these assessments, a planned three to six patients were recruited in a dose-escalation cohort (cohort 2) in which they received a single dose of adavosertib $225 \mathrm{mg}$ in cycle 0 , followed $5 \pm 2$ days later by adavosertib $225 \mathrm{mg}$ bid for 2.5 days in combination with paclitaxel plus carboplatin in subsequent 
cycles (A225PC). If two or more patients experienced a DLT in a group of up to six patients, irrespective of the number of patients enrolled, the dose was considered not tolerated and recruitment to the cohort and dose escalation ceased. A lower intermediary dose (de-escalation) could be considered in order to better define the combination recommended dose for further clinical evaluation (Supplementary Material).

After six cycles, patients in cohorts 1 and 2 could continue adavosertib monotherapy, whereas patients in cohort 1a continued combination therapy until disease progression or unacceptable toxicity.

An additional three to six patients could be recruited to the cohort in which the recommended combination dose was defined for further evaluation of the safety, tolerability, and pharmacokinetics of adavosertib.

Pre-medication with antiemetics (excluding aprepitant) was allowed.

The study was performed in accordance with the Declaration of Helsinki, Good Clinical Practice, applicable regulatory requirements, and the AstraZeneca policy on bioethics [11]. The institutional review boards or independent ethics committees of all investigational sites approved the protocol, and all patients provided written, informed consent.

\subsection{Study Objectives}

The primary objective was to assess the safety and tolerability of adavosertib in combination with carboplatin or paclitaxel plus carboplatin. Secondary objectives were to characterize the pharmacokinetic profiles of adavosertib (after single dosing and at steady state after multiple dosing with carboplatin or paclitaxel plus carboplatin), paclitaxel (in combination with adavosertib and carboplatin) and carboplatin (in combination with adavosertib or adavosertib and paclitaxel). An additional secondary objective was to obtain a preliminary assessment of the antitumor activity of adavosertib in combination with carboplatin alone or with paclitaxel plus carboplatin.

\subsection{Assessments}

Adverse events (AEs) were monitored throughout the study until the end of the follow-up period ( $28 \pm 7$ days after study treatment was discontinued or completed) and were graded using the National Cancer Institute's Common Terminology Criteria for Adverse Events (v4.03). Laboratory parameters, electrocardiogram (ECG) changes, and vital signs were also monitored. Hematology laboratory evaluations were performed at screening, on day 1 of cycle 0 , and on days 1,8 , and 15 for cycles 1 and beyond. Coagulation laboratory evaluations were performed at screening and on day 1 of every
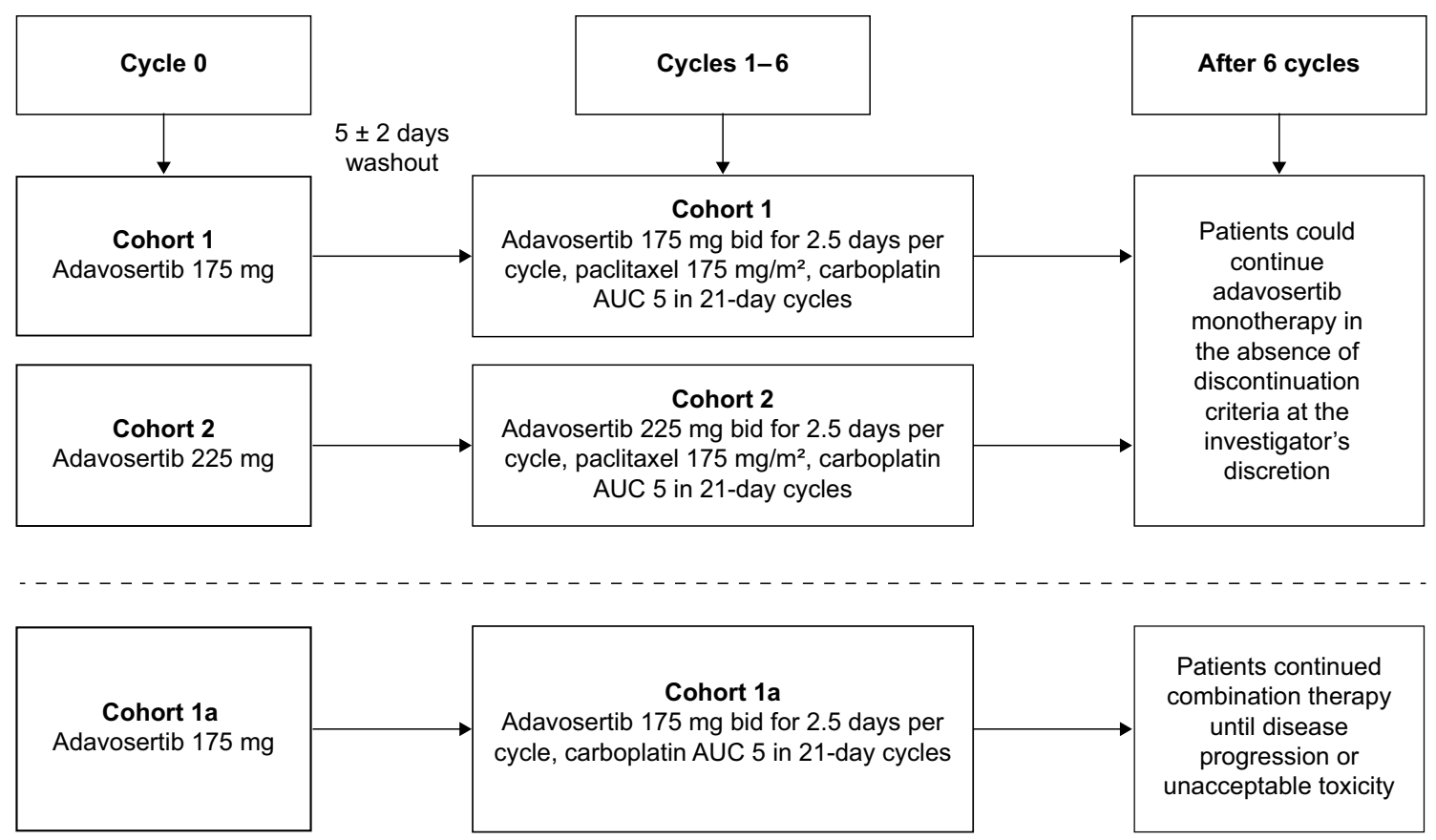

Fig. 1 Treatment cohorts. Patients were initially recruited into cohorts 1 and 1a, with 3-6 patients planned for each cohort. Based on safety and dose-limiting toxicity assessments, a planned 3-6 patients were then recruited in a dose-escalation cohort (cohort 2). An addi- tional 3-6 patients could be recruited to the cohort in which the recommended combination dose was defined for further evaluation of the safety, tolerability and pharmacokinetics of adavosertib. bid twice daily 
cycle. Chemistry laboratory evaluations were performed at screening, on day 1 of cycle 0 , on days 1 and 15 of cycle 1 , and on day 1 of each additional 21-day cycle.

Blood samples for pharmacokinetic analysis of adavosertib were collected pre-dose and up to $8 \mathrm{~h}$ post-dose on: cycle 0 , day 1 following a single dose; cycle 1 , day 1 following a single dose in combination with chemotherapy; and cycle 1, day 3 following multiple doses in combination with chemotherapy (Supplementary Material). Additional samples were collected pre-dose at day 3 of odd-numbered cycles. Plasma concentrations of adavosertib were determined by using a validated liquid chromatography-tandem mass spectrometry assay with a lower limit of quantitation of $2 \mathrm{ng} / \mathrm{mL}$.

Blood samples for pharmacokinetic analysis of paclitaxel and carboplatin were collected pre-dose and up to $8 \mathrm{~h}$ after the start of infusion at cycle 1, day 1 (Supplementary Material).

Response was evaluated according to RECIST v1.1 at screening and then at cycle 1, day 1 and every 6 weeks thereafter (at Japanese sites, an additional assessment occurred at cycle 2, day 1 ).

\subsection{Statistical Analysis}

To assess the tolerability of adavosertib in combination with chemotherapy, cohorts of at least six evaluable patients were required, with an expected sample size of approximately 18 patients per combination therapy.

The safety analysis set comprised all patients who received at least one dose of adavosertib, paclitaxel, or carboplatin, the pharmacokinetic analysis set comprised dosed patients for whom an adequate pharmacokinetic profile had been obtained, and the tumor-response analysis set comprised dosed patients with measurable disease at baseline.

Safety assessments, pharmacokinetic parameters, and tumor response were analyzed by descriptive statistics.

\section{Results}

\subsection{Patient Characteristics}

Seven, six, and six patients were treated in cohorts 1,2 , and $1 \mathrm{a}$, respectively. One patient in cohort 1 was recruited in error (without measurable disease); data from this patient were used in the safety and pharmacokinetic analyses but excluded from the tumor response analyses. One patient in cohort 2 and one in cohort 1a were still receiving study treatment at data cut-off (14 December 2016); the patient in cohort 2 was receiving adavosertib monotherapy. All patients were of Asian descent; patient baseline characteristics are shown in Table 1.
Exposure to adavosertib, paclitaxel, and carboplatin is summarized in Supplementary Table S1. There were no adavosertib dose reductions during cycle 0 or 1 , and no paclitaxel or carboplatin dose reductions during cycle 1 . After cycle 1 , adavosertib dose reductions occurred in three of six patients $(50.0 \%)$ from cohort 2 , paclitaxel dose reductions occurred in two of seven patients (28.6\%) from cohort 1 and three of six patients (50.0\%) from cohort 2, and carboplatin dose reductions occurred in one of seven patients (14.3\%) from cohort 1, four of six patients (66.7\%) from cohort 2 , and one of six patients (16.7\%) from cohort 1a.

\subsection{Safety and Tolerability}

DLTs (grade 4 decreased platelet count) occurred in one patient each in cohorts 1 and $1 \mathrm{a}$. Two patients in cohort 2 experienced DLTs (grade 4 sepsis and grade 5 acute respiratory distress syndrome).

Treatment-emergent adverse events (TEAEs) reported in at least one patient after a single dose of adavosertib were nausea (three of 19 [15.8\%] patients), constipation (one [5.3\%] patient), diarrhea (one [5.3\%] patient), and hypersensitivity (one [5.3\%] patient). All of these TEAEs were grade 1 and none occurred in more than one patient in each cohort.

With combination therapy, the most commonly reported TEAEs (all grades) included nausea, vomiting, anemia, diarrhea, and decreased white blood cell (WBC) count (Table 2). Hematologic AEs were the most commonly reported grade $\geq 3$ TEAEs (Table 2). The incidence of TEAEs (all grades and grade $\geq 3$ ) was generally highest in cohort 2 and lowest in cohort 1a. The small number of patients in each cohort should be considered when interpreting the incidence of TEAEs.

The most commonly occurring TEAEs (incidence of $>50 \%$ in any cohort) considered by the investigator as being potentially related to treatment were nausea $(85.7 \%$, $83.3 \%$, and $50.0 \%$ of patients in cohorts 1,2 , and $1 \mathrm{a}$, respectively), diarrhea $(71.4 \%, 83.3 \%$, and $33.3 \%$ ), vomiting $(71.4 \%, 66.7 \%$, and $33.3 \%)$, anemia $(71.4 \%, 66.7 \%$, and $33.3 \%)$, decreased WBC count $(71.4 \%, 83.3 \%$, and $50.0 \%)$, and decreased platelet count $(57.1 \%, 50.0 \%$, and $33.3 \%)$.

Fatal TEAEs were reported in one patient each in cohorts 1 and 2 (Table 2). The patient from cohort 1 had abnormal hepatic function resulting in liver failure and death. This patient had advanced head and neck cancer with tumors in the liver and a history of hepatomegaly. Extensive liver metastases were documented on day 15 of cycle 5, and the patient died on day 19 of cycle 5 . The investigator attributed death "almost certainly" to disease progression and considered the event to be unrelated to adavosertib. The patient from cohort 2 had acute respiratory distress syndrome in association with interstitial pneumonia, which was considered by the investigator to be possibly related to adavosertib 
Table 1 Patient baseline characteristics

\begin{tabular}{|c|c|c|c|}
\hline & $\begin{array}{l}\text { Cohort } 1 \text { (A175PC) } \\
N=7\end{array}$ & $\begin{array}{l}\text { Cohort } 2 \text { (A225PC) } \\
N=6\end{array}$ & $\begin{array}{l}\text { Cohort 1a (A175C) } \\
N=6\end{array}$ \\
\hline \multicolumn{4}{|l|}{ Age, years } \\
\hline Median (min, max) & $55.0(46,62)$ & $57.0(34,66)$ & $49.5(19,56)$ \\
\hline \multicolumn{4}{|l|}{ Race, $n(\%)$} \\
\hline Asian & $7(100)$ & $6(100)$ & $6(100)$ \\
\hline \multicolumn{4}{|l|}{ Sex, $n(\%)$} \\
\hline Male & $4(57.1)$ & $1(16.7)$ & $3(50.0)$ \\
\hline Female & $3(42.9)$ & $5(83.3)$ & $3(50.0)$ \\
\hline \multicolumn{4}{|l|}{ Type of cancer, $n(\%)$} \\
\hline Breast & 0 & $3(50.0)$ & $1(16.7)$ \\
\hline Head and neck ${ }^{\mathrm{a}}$ & $2(28.6)$ & 0 & 0 \\
\hline Cervix & $1(14.3)$ & 0 & $1(16.7)$ \\
\hline Ovary & $1(14.3)$ & $1(16.7)$ & 0 \\
\hline Uterus & $1(14.3)$ & $1(16.7)$ & 0 \\
\hline Other $^{b}$ & $2(28.6)$ & $1(16.7)$ & $4(66.7)$ \\
\hline \multicolumn{4}{|c|}{ Time since metastatic diagnosis, years } \\
\hline Median (min, max) & $1.61(0,2.5)$ & $4.60(2.2,9.3)$ & $1.87(0.9,4.9)$ \\
\hline \multicolumn{4}{|c|}{ Prior chemotherapy regimens, $n(\%)$} \\
\hline 1 & 0 & $1(16.7)$ & 0 \\
\hline 2 & $2(28.6)$ & 0 & $1(16.7)$ \\
\hline 3 & 0 & $1(16.7)$ & $1(16.7)$ \\
\hline 4 & $1(14.3)$ & 0 & 0 \\
\hline$\geq 5$ & $4(57.1)$ & $4(66.7)$ & $4(66.7)$ \\
\hline \multicolumn{4}{|c|}{ Best response to prior cancer therapy, $n(\%)$} \\
\hline Partial response & $3(42.9)$ & $2(33.3)$ & $3(50.0)$ \\
\hline Stable disease & $4(57.1)$ & $3(50.0)$ & $2(33.3)$ \\
\hline Disease progression & 0 & $1(16.7)$ & $1(16.7)$ \\
\hline
\end{tabular}

A175C adavosertib $175 \mathrm{mg}+$ carboplatin, $A 175 P C$ adavosertib $175 \mathrm{mg}+$ paclitaxel + carboplatin, $A 225 P C$ adavosertib $225 \mathrm{mg}+$ paclitaxel + carboplatin

${ }^{a}$ Including cancer of the nasopharynx, larynx, and trachea

${ }^{\mathrm{b}}$ Including stomach cancer $(n=1)$, pancreatic cancer $(n=1)$, skin/soft tissue cancer $(n=1)$, lung cancer $(n=1)$, thymic cancer $(n=2)$, and gallbladder cancer $(n=1)$

and paclitaxel. This patient had advanced lung cancer with previous tomotherapy to the lung and prior left upper lung lobectomy and was hospitalized and died on day 5 of cycle 1.

Serious TEAEs were reported in seven patients in cohorts 1 and 2 (Table 2). Six patients had serious AEs considered to be causally related to adavosertib: nausea and vomiting in one patient (cohort 1); decreased platelet count in one patient (cohort 1); neutropenia in one patient (cohort 2); febrile neutropenia in one patient each in cohorts 1 and 2; and diarrhea, acute respiratory distress syndrome, and interstitial pneumonia in one patient (cohort 2).

TEAEs resulted in discontinuation of adavosertib in one patient (14.3\%) from cohort 1 (micturition urgency, nausea, and vomiting) and two patients (33.3\%) from cohort 2 (febrile neutropenia in one patient and acute respiratory distress syndrome, interstitial pneumonia, and diarrhea in a second patient). Paclitaxel was discontinued because of TEAEs in one patient (14.3\%) from cohort 1 and two patients $(33.3 \%)$ from cohort 2, and carboplatin was discontinued because of TEAEs in two patients (33.3\%) from cohort 2.

No trends were observed in vital signs over time, and there were no clinically important changes in ECG recordings.

\subsection{Pharmacokinetic Profile}

Adavosertib was steadily absorbed, with a median time to maximum plasma concentration $\left(t_{\max }\right)$ of $2.02-4.04 \mathrm{~h}$, and then slowly eliminated (Fig. 2 and Table 3 ). After reaching the maximum plasma concentration $\left(C_{\max }\right)$, adavosertib concentrations 
Table 2 Adverse events occurring with adavosertib and chemotherapy

\begin{tabular}{|c|c|c|c|}
\hline $\begin{array}{l}\text { Patients experiencing TEAEs }{ }^{\mathrm{a}} \text {, } \\
n(\%)\end{array}$ & $\begin{array}{l}\text { Cohort } 1 \\
\text { (A175PC) } \\
N=7\end{array}$ & $\begin{array}{l}\text { Cohort } 2 \\
\text { (A225PC) } \\
N=6\end{array}$ & $\begin{array}{l}\text { Cohort 1a } \\
\text { (A175C) } \\
N=6\end{array}$ \\
\hline TEAEs $^{\mathrm{b}}$ & $6(85.7)$ & $6(100.0)$ & $6(100.0)$ \\
\hline Nausea & $6(85.7)$ & $5(83.3)$ & $5(83.3)$ \\
\hline Vomiting & $6(85.7)$ & $5(83.3)$ & $4(66.7)$ \\
\hline Anemia & $6(85.7)$ & $5(83.3)$ & $4(66.7)$ \\
\hline Diarrhea & $5(71.4)$ & $5(83.3)$ & $3(50.0)$ \\
\hline WBC count decreased & $5(71.4)$ & $5(83.3)$ & $3(50.0)$ \\
\hline Decreased appetite & $4(57.1)$ & $2(33.3)$ & $4(66.7)$ \\
\hline Platelet count decreased $^{c}$ & $4(57.1)$ & $3(50.0)$ & $3(50.0)$ \\
\hline Neutrophil count decreased ${ }^{\mathrm{d}}$ & $3(42.9)$ & $3(50.0)$ & $4(66.7)$ \\
\hline Pyrexia & $3(42.9)$ & $4(66.7)$ & $1(16.7)$ \\
\hline Neutropenia $^{\mathrm{d}}$ & $3(42.9)$ & $2(33.3)$ & 0 \\
\hline Constipation & $2(28.6)$ & $3(50.0)$ & 0 \\
\hline Proctalgia & $1(14.3)$ & $3(50.0)$ & $1(16.7)$ \\
\hline Grade $\geq 3$ TEAEs $^{\mathrm{e}}$ & $6(85.7)$ & $6(100.0)$ & $4(66.7)$ \\
\hline Anemia & $4(57.1)$ & $5(83.3)$ & $2(33.3)$ \\
\hline WBC count decreased & $5(71.4)$ & $5(83.3)$ & $1(16.7)$ \\
\hline Platelet count decreased $^{\mathrm{f}}$ & $4(57.1)$ & $3(50.0)$ & $2(33.3)$ \\
\hline Neutrophil count decreased ${ }^{\mathrm{d}}$ & $3(42.9)$ & $3(50.0)$ & $2(33.3)$ \\
\hline Neutropenia $^{\mathrm{d}}$ & $3(42.9)$ & $2(33.3)$ & 0 \\
\hline Thrombocytopenia $^{\mathrm{f}}$ & $1(14.3)$ & $2(33.3)$ & $1(16.7)$ \\
\hline Febrile neutropenia & $1(14.3)$ & $2(33.3)$ & 0 \\
\hline Diarrhea & $1(14.3)$ & $2(33.3)$ & 0 \\
\hline Hypophosphatemia & $1(14.3)$ & $2(33.3)$ & 0 \\
\hline Sepsis & 0 & $2(33.3)$ & 0 \\
\hline Treatment-related TEAEs & $6(85.7)$ & $6(100.0)$ & $4(66.7)$ \\
\hline Serious TEAEs & $3(42.9)$ & $4(66.7)$ & 0 \\
\hline Fatal TEAEs & $1(14.3)$ & $1(16.7)$ & 0 \\
\hline
\end{tabular}

A175C adavosertib $175 \mathrm{mg}+$ carboplatin, $A 175 P C$ adavosertib $175 \mathrm{mg}+$ paclitaxel + carboplatin, A225PC adavosertib $225 \mathrm{mg}$ + paclitaxel + carboplatin, TEAE treatment-emergent adverse event, $W B C$ white blood cell

${ }^{a}$ TEAE defined as any AE that occurred after administration of the first dose of study drug and through 28 days after the last dose of study drug, or any event that was present at baseline and continued after the first dose of study drug but worsened in intensity

${ }^{b}$ TEAEs occurring with an incidence of $>40 \%$ in any cohort

${ }^{\mathrm{c}}$ None of the patients reported to have a decreased platelet count were also reported to have thrombocytopenia $(n=1[14.3 \%]$ in cohort 1 ; $n=2[33.3 \%]$ in cohort $2 ; \mathrm{n}=1[16.7 \%]$ in cohort 1a)

${ }^{\mathrm{d}}$ None of the patients reported to have a decreased neutrophil count were also reported to have neutropenia

${ }^{\mathrm{e}}$ Grade $\geq 3$ TEAEs occurring with an incidence of $>30 \%$ in any cohort

${ }^{\mathrm{f}}$ None of the patients reported to have a decreased platelet count were also reported to have thrombocytopenia

remained relatively stable over the 8-h sampling period, with the geometric mean plasma concentration at $8 \mathrm{~h}\left(C_{8 \mathrm{~h}}\right)$ being $53-77 \%$ of the corresponding geometric mean $C_{\max }$ values.

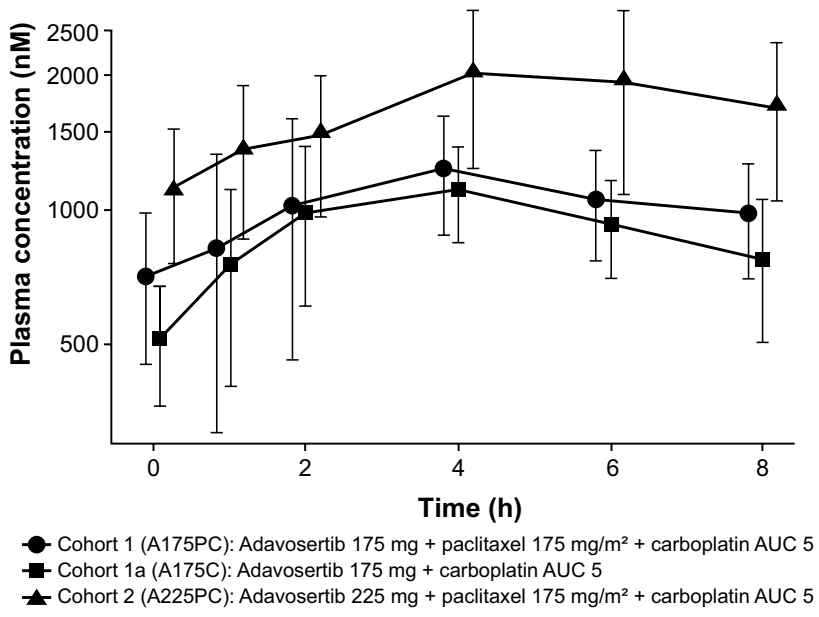

Fig. 2 Semi-logarithmic plot showing the geometric mean ( \pm standard deviation) plasma concentration-time profile for adavosertib on cycle 1 , day $3 *$.bid twice daily; *Multiple doses of adavosertib $175 \mathrm{mg}$ bid with paclitaxel plus carboplatin (cohort 1), adavosertib $225 \mathrm{mg}$ bid with paclitaxel plus carboplatin (cohort 2), or adavosertib $175 \mathrm{mg}$ bid with carboplatin (cohort 1a)

Systemic exposure to adavosertib increased in a slightly greater than dose-proportional manner as the adavosertib dose increased from 175 to $225 \mathrm{mg}$ bid (Table 3). A 1.3-fold increase in adavosertib dose resulted in a 1.3- to 1.8-fold increase in geometric mean area under the plasma concentration-time curve from time zero until the last quantifiable concentration $\left(\mathrm{AUC}_{0-t}\right)$.

Adavosertib accumulated in plasma after administration of multiple doses, with a geometric mean (coefficient of variation) accumulation ratio of $2.1(38 \%)$ in cohort $1,2.7$ (17\%) in cohort 2, and $2.3(15 \%)$ in cohort $1 \mathrm{a}$.

No drug-drug interactions were observed for any of the agents administered in this study (Table 3 and Supplementary Tables S2 and S3).

\subsection{Antitumor Activity}

The best overall response observed was a partial response in one of six evaluable patients (16.7\%) in both cohorts 1 and $1 \mathrm{a}$, and in three of six evaluable patients $(50.0 \%)$ in cohort 2 (Table 4). The overall median duration of response in these five patients was 20.7 weeks (95\% confidence interval 18.1, not evaluable; Kaplan-Meier estimate). Stable disease occurred in two of six evaluable patients (33.3\%) in each cohort.

\section{Discussion}

Results of Phase II studies indicate that adavosertib sensitizes patients to chemotherapy [7, 12]. Adavosertib showed efficacy when combined with carboplatin in women with 
Table 3 Single-dose and multiple-dose pharmacokinetics of adavosertib

\begin{tabular}{|c|c|c|c|c|c|c|c|c|c|}
\hline \multirow[t]{2}{*}{ Parameter } & \multicolumn{3}{|c|}{ Cycle 0 , day $1^{\mathrm{a}}$} & \multicolumn{3}{|c|}{ Cycle 1 , day $1^{\mathrm{b}}$} & \multicolumn{3}{|c|}{ Cycle 1 , day $3^{\mathrm{c}}$} \\
\hline & $\begin{array}{l}\text { Cohort } 1 \\
(N=7)^{\mathrm{d}}\end{array}$ & $\begin{array}{l}\text { Cohort } 2 \\
(N=6)\end{array}$ & $\begin{array}{l}\text { Cohort 1a } \\
(N=5)^{\mathrm{e}}\end{array}$ & $\begin{array}{l}\text { Cohort } 1 \\
(N=6)^{\mathrm{f}}\end{array}$ & $\begin{array}{l}\text { Cohort } 2 \\
(N=6)\end{array}$ & $\begin{array}{l}\text { Cohort 1a } \\
(N=6)^{\mathrm{f}}\end{array}$ & $\begin{array}{l}\text { Cohort } 1 \\
(N=6)\end{array}$ & $\begin{array}{l}\text { Cohort } 2 \\
(N=6)^{\mathrm{f}}\end{array}$ & $\begin{array}{l}\text { Cohort 1a } \\
(N=6)\end{array}$ \\
\hline \multicolumn{10}{|l|}{$\begin{array}{c}\mathrm{AUC}_{0-t}, \\
\mathrm{nM} \cdot \mathrm{h}\end{array}$} \\
\hline Gmean & 3521 & 5331 & 3387 & 4191 & 5606 & 2902 & 8300 & 14,870 & 7154 \\
\hline $\mathrm{CV}, \%$ & 44.91 & 37.42 & 12.54 & 34.89 & 20.02 & 33.21 & 32.85 & 34.05 & 32.29 \\
\hline \multicolumn{10}{|l|}{$C_{\max }, \mathrm{nM}$} \\
\hline Gmean & 689.1 & 1066 & 649.2 & 705.4 & 1133 & 654.8 & 1271 & 2289 & 1129 \\
\hline $\mathrm{CV}, \%$ & 51.79 & 38 & 10.74 & 28.03 & 16.3 & 32.27 & 30.52 & 32.82 & 25.51 \\
\hline \multicolumn{10}{|l|}{$C_{8 \mathrm{~h}}, \mathrm{nM}$} \\
\hline Gmean & 370.1 & 612 & 343.5 & 446.6 & 805.9 & 378.2 & 982 & 1700 & 774.6 \\
\hline $\mathrm{CV}, \%$ & 29.15 & 36.6 & 29.97 & 27.83 & 27.5 & 27.61 & 30.17 & 37.6 & 32.96 \\
\hline \multicolumn{10}{|l|}{$t_{\max }, \mathrm{h}$} \\
\hline Median & 2.02 & 3.96 & 3.95 & 4.00 & 4.04 & 4.04 & 4.00 & 4.04 & 3.09 \\
\hline Min, $\max$ & $1.00,8.00$ & $1.00,5.87$ & $2.00,8.00$ & $0.95,8.02$ & $3.98,6.00$ & $4.00,8.00$ & $2.05,4.08$ & $1.00,7.95$ & $1.95,4.08$ \\
\hline \multicolumn{10}{|l|}{$t_{\text {last }}, \mathrm{h}$} \\
\hline Median & 8.00 & 8.00 & 7.98 & 8.01 & 8.03 & 7.65 & 7.96 & 7.98 & 7.98 \\
\hline Min, $\max$ & $7.83,8.00$ & $7.97,8.02$ & $7.20,8.00$ & $7.92,8.17$ & $7.95,8.08$ & $7.20,8.00$ & $7.85,8.00$ & $7.85,8.15$ & $7.20,8.17$ \\
\hline
\end{tabular}

$A U C_{0-t}$ area under the plasma concentration-time curve from time zero until the last quantifiable concentration, $C_{8 h}$ plasma concentration at $8 \mathrm{~h}$, $C_{\max }$ maximum plasma concentration, $C V$ coefficient of variation, Gmean geometric mean, $t_{\text {last }}$ time to the last quantifiable concentration, $t_{\max }$ time to maximum plasma concentration

${ }^{a}$ Cycle 0, day 1: single dose of adavosertib $175 \mathrm{mg}$ (cohorts 1 and 1a) or adavosertib $225 \mathrm{mg}$ (cohort 2)

${ }^{\mathrm{b}}$ Cycle 1, day 1: single dose of adavosertib $175 \mathrm{mg}$ with paclitaxel plus carboplatin (cohort 1), single dose of adavosertib $225 \mathrm{mg}$ with paclitaxel plus carboplatin (cohort 2), or single dose of adavosertib $175 \mathrm{mg}$ with carboplatin (cohort 1a)

${ }^{\mathrm{c}}$ Cycle 1, day 3: multiple doses of adavosertib $175 \mathrm{mg}$ with paclitaxel plus carboplatin (cohort 1), multiple doses of adavosertib $225 \mathrm{mg}$ with paclitaxel plus carboplatin (cohort 2), or multiple doses of adavosertib $175 \mathrm{mg}$ with carboplatin (cohort 1a)

${ }^{\mathrm{d}} \mathrm{N}=6$ for $\mathrm{AUC}_{0-\mathrm{t}}$ and $\mathrm{C}_{\max }$

${ }^{\mathrm{e}} \mathrm{N}=4$ for $\mathrm{AUC}_{0-\mathrm{t}}$ and $\mathrm{C}_{\text {max }}$

${ }^{\mathrm{f}} \mathrm{N}=5$ for $\mathrm{AUC}_{0-\mathrm{t}}$ and $\mathrm{C}_{\max }$

TP53-mutated ovarian cancer refractory or resistant to firstline platinum-based chemotherapy, with sustained responses of over 30 months seen in two patients [7]. Adavosertib also showed activity in combination with single-agent chemotherapy (carboplatin, paclitaxel, gemcitabine, or pegylated liposomal doxorubicin) in women with primary platinumresistant ovarian cancer [12]. Results of these studies support a role for adavosertib in tumors that respond inadequately to chemotherapy.

This Phase Ib study investigated the optimal adavosertib dosage in Asian patients. Various factors, including race, affect drug pharmacokinetics. Differences observed between Western and Asian patients in exposure to some drugs may be attributable to factors such as body weight and drug metabolism [13, 14]. The recommended Phase II regimen of adavosertib for use in combination with paclitaxel and carboplatin in Western patients is $225 \mathrm{mg}$ bid for 2.5 days [10].

This study investigated regimens of adavosertib $175 \mathrm{mg}$ bid for 2.5 days in combination with carboplatin alone or with paclitaxel plus carboplatin, or adavosertib $225 \mathrm{mg}$ bid for 2.5 days in combination with paclitaxel plus carboplatin. Pharmacokinetic analyses indicated that adavosertib was steadily absorbed and then slowly eliminated, and that coadministration of paclitaxel and carboplatin had no apparent effect on the pharmacokinetics of adavosertib. It should be noted that the terminal elimination phase of adavosertib could not be fully characterized because blood sampling was only conducted up to $8 \mathrm{~h}$ post-dose. Data suggest that exposure of adavosertib was 30-45\% higher in Asian patients in this study than in Western patients [8], which explains, at least in part, the tolerability findings. Additional investigation to better understand these differences is ongoing.

Adavosertib $175 \mathrm{mg}$ bid was considered tolerable when administered for 2.5 days per 21-day cycle in combination with paclitaxel plus carboplatin, or with only carboplatin, in Asian patients. However, adavosertib $225 \mathrm{mg}$ bid for 2.5 days administered in combination with paclitaxel plus carboplatin was not considered tolerable, with two patients 
Table 4 Antitumor activity of adavosertib in combination with chemotherapy

\begin{tabular}{lccc}
\hline & $\begin{array}{l}\text { Cohort 1 (A175PC) } \\
N=6\end{array}$ & $\begin{array}{l}\text { Cohort 2 (A225PC) } \\
N=6\end{array}$ & $\begin{array}{c}\text { Cohort 1a (A175C) } \\
N=6\end{array}$ \\
\hline $\begin{array}{l}\text { Best overall response, } n(\%) \\
\text { Complete response }\end{array}$ & $0(0)$ & $0(0)$ & $0(0)$ \\
Partial response & $1(16.7)$ & $3(50.0)$ & $1(16.7)$ \\
Stable disease & $2(33.3)$ & $2(33.3)$ & $2(33.3)$ \\
Progressive disease & $2(33.3)$ & $0(0)$ & $1(16.7)$ \\
Not evaluable & $1(16.7)$ & $1(16.7)$ & $2(33.3)$ \\
Objective response $^{\mathrm{a}}, n(\%)$ & $1(16.7)$ & $3(50.0)$ & $1(16.7)$ \\
Clinical benefit $^{\mathrm{b}}, n(\%)$ & $3(50.0)$ & $5(83.3)$ & $3(50.0)$ \\
Median duration of response $^{\mathrm{c}}$, weeks $(95 \% \mathrm{CI})$ & $18.1(\mathrm{NE}, \mathrm{NE})$ & $20.7(\mathrm{NE}, \mathrm{NE})$ & $\mathrm{NE}(\mathrm{NE}, \mathrm{NE})$ \\
\hline
\end{tabular}

$A 175 C$ adavosertib $175 \mathrm{mg}+$ carboplatin, $A 175 P C$ adavosertib $175 \mathrm{mg}+$ paclitaxel + carboplatin, $A 225 P C$ adavosertib $225 \mathrm{mg}+$ paclitaxel + carboplatin, $C I$ confidence interval, $N E$ not evaluable

${ }^{a}$ Confirmed complete response or partial response

${ }^{\mathrm{b}}$ Confirmed complete response, partial response, or stable disease

${ }^{\mathrm{c}}$ Kaplan-Meier estimates

experiencing DLTs (grade 4 sepsis and grade 5 acute respiratory distress syndrome). The event of acute respiratory distress syndrome was considered by the investigator to be possibly related to adavosertib and paclitaxel; other potential contributing factors included the patient's underlying disease (metastatic lung cancer), prior chemotherapy and radiotherapy, and possible infection. Dose escalation in the cohort receiving adavosertib with only carboplatin was not evaluated in this study.

The tolerability profile of adavosertib $175 \mathrm{mg}$ bid for 2.5 days plus chemotherapy in this study was generally similar to that reported in Western women who received adavosertib $225 \mathrm{mg}$ bid for 2.5 days plus chemotherapy [7, 8]. For example, the most commonly reported TEAEs (all grades) were fatigue (87\% of patients), nausea (78\%), diarrhea $(70 \%)$, thrombocytopenia $(70 \%)$, and anemia $(61 \%)$ in women with ovarian cancer refractory or resistant to firstline platinum-based chemotherapy who received adavosertib plus carboplatin in the PN009 study [7]. In women with platinum-sensitive ovarian cancer who received adavosertib in combination with paclitaxel and carboplatin in the PNO04 study, the most commonly reported AEs (all grades) were nausea $(78 \%)$, diarrhea (75\%), vomiting (63\%), alopecia (54\%), and fatigue (54\%) [8]. In this study, fatigue was reported in $28.6 \%, 16.7 \%$, and $33.3 \%$ of patients in cohorts 1,2 , and $1 \mathrm{a}$, respectively.

Preliminary assessment of adavosertib in combination with paclitaxel plus carboplatin or with only carboplatin indicated antitumor activity in heavily pre-treated Asian patients with advanced solid tumors. However, the small number of patients in each treatment group meant that no firm conclusions could be drawn regarding differences in tumor response endpoints. In addition to small patient numbers, interpretation of the duration of response is limited by the fact that patients were not followed up for progression after discontinuation of treatment.

Although adavosertib $225 \mathrm{mg}$ bid for 2.5 days demonstrated antitumor activity, based on the tolerability findings, adavosertib $175 \mathrm{mg}$ bid for 2.5 days was chosen as the recommended Phase II dose in combination with paclitaxel and carboplatin in Asian patients.

In terms of other adavosertib trials, results of Phase I studies indicate that combination therapy with adavosertib plus olaparib [15] or durvalumab [16] had a manageable tolerability profile in patients with advanced solid tumors. A Phase I study also demonstrated that adavosertib in combination with neoadjuvant docetaxel and cisplatin had an acceptable tolerability profile in patients with advanced head and neck squamous cell carcinoma [17]. Ongoing studies in Asian patients include a Phase II study (NCT02593019) evaluating the efficacy of adavosertib monotherapy in Asian patients with relapsed small-cell lung cancer.

\section{Conclusions}

In Asian patients, the recommended Phase II dose of adavosertib (175 mg bid for 2.5 days per 21-day cycle in combination with paclitaxel plus carboplatin) is lower than the recommended Phase II dose in Western patients, most likely reflecting the increased exposure of adavosertib in Asian versus Western patients. Preliminary assessment of adavosertib in combination with paclitaxel plus carboplatin or 
with only carboplatin indicated antitumor activity in Asian patients with advanced solid tumors.

Acknowledgements Medical writing assistance was provided by Gillian Keating, MBChB, from Mudskipper Business Ltd, funded by AstraZeneca. We thank David White from INC Research, UK, who contributed to data analysis.

Authors' contributions Acquisition of data: H Kato, P de Souza, S-W Kim, J Lickliter, Y Naito, K Park, Y-J Bang. Analysis and/or interpretation of data: H Kato, P de Souza, S-W Kim, J Lickliter, Y Naito, K Park, S Kumar, GM Mugundu, Y-J Bang. Writing, review and/or revision of the manuscript: H Kato, P de Souza, S-W Kim, J Lickliter, Y Naito, K Park, S Kumar, GM Mugundu, Y-J Bang.

\section{Compliance with Ethical Standards}

The study was performed in accordance with the Declaration of Helsinki, Good Clinical Practice, applicable regulatory requirements, and the AstraZeneca policy on bioethics [11]. The institutional review boards or independent ethics committees of all investigational sites approved the protocol, and all patients provided written, informed consent.

Funding This work was supported by AstraZeneca.

Conflict of interest P de Souza is a consultant for Noxopharm Australia Pty Ltd and BioSceptre Australia Pty Ltd. S-W Kim reports an advisory role for AstraZeneca, Lilly, Ono, and Boehringer Ingelheim and research funding from AstraZeneca, Lilly, and Boehringer Ingelheim. Y Naito reports a speakers' bureau role for AstraZeneca, Bayer, Chugai, Eisai, Eli Lilly, Fuji Film Toyama Chemical, Meiji Seika, Merck Serono, Nippon Kayaku, Novartis, Pfizer, Roche Diagnostics, and Taiho, and research funding from Roche Diagnostics. K Park reports an advisory role for AbbVie, Amgen, Astellas Pharma, AstraZeneca, Boehringer Ingelheim, BluePrint, BMS, Clovis Oncology, Daiichi-Sankyo, Eli Lilly, GSK, Hanmi, Incyte, Kyowa Hakko Kirin, LOXO, Merck KGaA, MSD, Novartis, Ono Pharmaceutical, and Roche and research funding from AstraZeneca and MSD. S Kumar and GM Mugundu are AstraZeneca employees and stock holders. Y-J Bang is a consultant and reports an advisory role for AstraZeneca, Novartis, Genentech/Roche, MSD, Merck Serono, Bayer, BMS, Eli Lilly, Taiho, Daiich-Sankyo, Astellas, BeiGene, GreenCross, Samyang Biopharm, Hanmi, and Genexine and institutional grants (for clinical trials) from AstraZeneca, Novartis, Genentech/Roche, MSD, Merck Serono, Bayer, BMS, GSK, Pfizer, Eli Lilly, Boehringer-Ingelheim, MacroGenics, Boston Biomedical, FivePrime, Curis, Taiho, Takeda, Ono, DaiichiSankyo, Astellas, BeiGene, GreenCross, CKD Pharma, and Genexine. No potential conflicts of interest were disclosed by the other authors.

Open Access This article is licensed under a Creative Commons Attribution-NonCommercial 4.0 International License, which permits any non-commercial use, sharing, adaptation, distribution and reproduction in any medium or format, as long as you give appropriate credit to the original author(s) and the source, provide a link to the Creative Commons licence, and indicate if changes were made. The images or other third party material in this article are included in the article's Creative Commons licence, unless indicated otherwise in a credit line to the material. If material is not included in the article's Creative Commons licence and your intended use is not permitted by statutory regulation or exceeds the permitted use, you will need to obtain permission directly from the copyright holder.To view a copy of this licence, visit http://creativecommons.org/licenses/by-nc/4.0/.

\section{References}

1. Aarts M, Sharpe R, Garcia-Murillas I, et al. Forced mitotic entry of S-phase cells as a therapeutic strategy induced by inhibition of WEE1. Cancer Discov. 2012;2:524-39.

2. Gerard C, Goldbeter A. The balance between cell cycle arrest and cell proliferation: control by the extracellular matrix and by contact inhibition. Interface Focus. 2014;4:20130075.

3. Leijen S, Beijnen JH, Schellens JH. Abrogation of the G2 checkpoint by inhibition of Wee-1 kinase results in sensitization of p53-deficient tumor cells to DNA-damaging agents. Curr Clin Pharmacol. 2010;5:186-91.

4. Do K, Doroshow JH, Kummar S. Wee1 kinase as a target for cancer therapy. Cell Cycle. 2013;12:3159-64.

5. Beck H, Nahse-Kumpf V, Larsen MS, et al. Cyclin-dependent kinase suppression by WEE1 kinase protects the genome through control of replication initiation and nucleotide consumption. Mol Cell Biol. 2012;32:4226-36.

6. Leijen S, van Geel RMJM, Pavlik AC, et al. Phase I study evaluating WEE1 inhibitor AZD1775 as monotherapy and in combination with gemcitabine, cisplatin, or carboplatin in patients with advanced solid tumors. J Clin Oncol. 2016;34:4371-80.

7. Leijen S, van Geel RMJM, Sonke GS, et al. Phase II study of WEE1 inhibitor AZD1775 plus carboplatin in patients with TP53mutated ovarian cancer refractory or resistant to first-line therapy within 3 months. J Clin Oncol. 2016;34:4354-61.

8. Oza AM, Weberpals JI, Provencher DM, et al. An international, biomarker-directed, randomized, phase II trial of AZD1775 plus paclitaxel and carboplatin $(\mathrm{P} / \mathrm{C})$ for the treatment of women with platinum-sensitive, TP53-mutant ovarian cancer. J Clin Oncol. 2015;33(15 Suppl):abst 5506.

9. Do K, Wilsker D, Ji J, et al. Phase I study of single-agent AZD1775 (MK-1775), a Wee1 kinase inhibitor, in patients with refractory solid tumors. J Clin Oncol. 2015;33:3409-15.

10. Brana I, Moore KN, Shapira-Frommer R, et al. Targeting p53 mutant ovarian cancer: Phase I results of the WEE1 inhibitor MK-1775 with carboplatin plus paclitaxel in patients (pts) with platinum-sensitive, p53-mutant ovarian cancer (OC). J Clin Oncol. 2013;31(15 Suppl):abst 5518.

11. AstraZeneca. Global policy: bioethics. 2016. https://www.astra zeneca.com/content/dam/az/PDF/Sustainability/code-of-ethic s-2018/AZ\%20Code\%20of\%20Ethics\%20-\%20English.pdf. Accessed 03 Feb 2020

12. Moore KN, Chambers SK, Hamilton EP, et al. Adavosertib with chemotherapy (CT) in patients (pts) with platinum-resistant ovarian cancer (PROC): An open-label, four-arm, phase II study. J Clin Oncol. 2019;37(Suppl):abst 5513.

13. Wang E, Nickens DJ, Bello A, et al. Clinical implications of the pharmacokinetics of crizotinib in populations of patients with non-small cell lung cancer. Clin Cancer Res. 2016;22:5722-8.

14. Wu HF, Hristeva N, Chang J, et al. Rosuvastatin pharmacokinetics in Asian and White subjects wild type for both OATP1B1 and BCRP under control and inhibited conditions. J Pharm Sci. 2017;106:2751-7.

15. Hamilton E, Falchook GS, Wang JS, et al. Phase Ib study of adavosertib in combination with olaparib in patients with refractory solid tumors: Dose escalation. Cancer Res. 2019;79(Suppl):abst CT025.

16. Patel MR, Falchook GS, Wang JS, et al. Open-label, multicenter, phase I study to assess safety and tolerability of advavosertib plus durvalumab in patients with advanced solid tumors. J Clin Oncol. 2019;37(Suppl):abst 2562.

17. Mendez E, Rodriguez CP, Kao MC, et al. A phase I clinical trial of AZD1775 in combination with neoadjuvant weekly docetaxel and cisplatin before definitive therapy in head and neck squamous cell carcinoma. Clin Cancer Res. 2018;24:2740-8. 


\section{Affiliations}

Hidenori Kato ${ }^{1}$ Paul de Souza ${ }^{2,10} \cdot$ Sang-We Kim ${ }^{3}$. Jason D. Lickliter ${ }^{4}$ - Yoichi Naito ${ }^{5} \cdot$ Keunchil Park $^{6}$. Sanjeev Kumar ${ }^{7}$. Ganesh M. Mugundu ${ }^{8} \cdot$ Yung-Jue Bang ${ }^{9}$

1 Division of Gynecologic Oncology, Hokkaido Cancer Center, Sapporo, Japan

2 Ingham Institute, Western Sydney University, Liverpool Hospital, Liverpool, NSW, Australia

3 Department of Oncology, Asan Medical Centre, Seoul, South Korea

4 Centre for Clinical Studies, Nucleus Network Limited, Melbourne, VIC, Australia

5 National Cancer Center Hospital East, Kashiwa, Japan

6 Samsung Medical Centre, Sungkyunkwan University School of Medicine, Seoul, South Korea

7 AstraZeneca, Cambridge, UK
8 Quantitative Clinical Pharmacology, Early Clinical Development, IMED Biotech Unit, AstraZeneca, Boston, MA, USA

9 Department of Internal Medicine, Seoul National University College of Medicine, 101 Daehak-ro, Jongno-gu, Seoul 03080, South Korea

10 Present Address: School of Medicine, University of Wollongong, Wollongong, NSW, Australia 